\title{
SÍNTESE DE ZEÓLITAS A PARTIR DE CINZAS VOLANTES EM SISTEMAS ABERTOS
}

\author{
P. F. PRADO ${ }^{1}$, M. NASCIMENTO ${ }^{2}$ e L. YOKOYAMA ${ }^{3}$ \\ ${ }^{1}$ Universidade Federal do Rio de Janeiro, Escola de Química, Programa de Pós Graduação em \\ Processos Químicos e Bioquímicos \\ ${ }^{2}$ Centro de Tecnologia Mineral, CETEM, Departamento de Tecnologias Limpas \\ ${ }^{3}$ Universidade Federal do Rio de Janeiro, Departamento de Processos Inorgânicos \\ E-mail para contato: patriciafprado@yahoo.com.br
}

\begin{abstract}
RESUMO - As zeólitas englobam um grande número de minerais naturais e sintéticos, cuja estrutura é formada basicamente por tetraedros de silício e alumínio unidos nos vértices por átomos de oxigênio, contendo canais e cavidades interconectadas de dimensões moleculares. Este trabalho tem como objetivo investigar a síntese de fases zeóliticas a partir da mulita, principal componente das cinzas volantes gerados pela queima de carvão mineral. Foram realizados ensaios preliminares de síntese de fases zeolíticas em sistemas abertos, a partir de cinzas de carvão. Os resultados mostram que a aplicação de temperatura ambiente nas concentrações e tempos testados resultou na síntese de uma zeólita $\mathrm{Na}-\mathrm{X}$ (tipo FAU) a partir da dissolução das cinzas. Uma pequena presença de philipsita também foi verificada em algumas amostras. Esses resultados mostram que a formação das zeólitas são influenciadas pelas variáveis de processo. Um aumento do tempo de síntese bem como um aumento da concentração de $\mathrm{NaOH}$ para a produção de fases zeolíticas resultam num aumento da capacidade de formação de zeólitas.
\end{abstract}

\section{INTRODUÇÃO}

Espécies tóxicas de metais pesados causam sérios danos ao ecossistema, e em decorrência deste fato, observa-se um aumento no número de pesquisas na área de processos para tratamento de efluentes. Muitos destes processos são baseados nas propriedades adsortivas ou de troca iônica de alguns materiais que imobilizam estas espécies de metais pesados. Recentemente, vários materiais de origem natural ou sintética, tais como: bagaço, cinzas de carvão, xisto, carbonatos, fosfatos e zeólitas, têm sido testados para avaliar a sua capacidade adsortiva. As zeólitas são comumente utilizadas para adsorção de metais pesados devido as suas propriedades físicas e químicas (estabilidade térmica, estrutura molecular definida e capacidade de troca iônica).

Um dos resíduos sólidos mais significativos em termos de volume no Brasil são as cinzas de carvão geradas nas termelétricas. A necessidade de retirar as cinzas das usinas a baixo custo faz com que se adotem práticas de disposição em áreas impróprias e sem medidas de proteção adequadas. A lixiviação de cinzas causa a contaminação de solos, das águas subterrâneas e superficiais por substâncias tóxicas. 
As principais aplicações das cinzas de carvão são nas fábricas de cimento e concreto, uso como fertilizantes, porém em pequena escala. Uma grande parte das cinzas são dispostas em lagoas de estabilização gerando grande impacto ambiental (Lorgouilloux et al.,2009). Uma das maneiras de reduzir os impactos ambientais decorrentes da disposição destes resíduos no meio ambiente consiste na ampliação das potencialidades de sua utilização. Uma alternativa de aproveitamento destes resíduos é a transformação das cinzas de carvão em adsorventes de baixo custo, capaz de remover substâncias tóxicas de águas contaminadas.

A síntese de zeólitas a partir de algumas fases minerais, como o carvão, por exemplo, tem sido investigada por diversos pesquisadores. Já é bem estabelecido que as cinzas de carvão compostas principalmente de fases como quartzo $\left(\mathrm{SiO}_{2}\right)$, mulita $\left(\mathrm{Al}_{4+2 \mathrm{x}} \mathrm{Si}_{2-2 \mathrm{x}} \mathrm{O}_{10-\mathrm{x}}\right)$, hematita $\left(\mathrm{Fe}_{2} \mathrm{O}_{3}\right)$ e magnetita $\left(\mathrm{Fe}_{3} \mathrm{O}_{4}\right)$ podem produzir zeólitas a partir de tratamento hidrotérmico alcalino (Dana, 1981).

A revisão da literatura mostra que as zeólitas podem ser produzidos por métodos hidrotérmicos utilizando cinzas volantes de carvão. No entanto sabe-se os materiais zeóliticos sintetizados a partir de cinzas volantes tem a sua eficiência como adsorventes para metais pesados influenciados pelas condições de síntese (Nascimento et al., 2009).

Na maioria dos casos, a influência dos seguintes parâmetros de síntese são estudadas, a concentração e natureza da base, o tempo e a temperatura de reação. A temperatura de síntese relatada em muitos artigos geralmente varia entre 80 e $200^{\circ} \mathrm{C}$, para as sínteses de zeólitas (Gross et al., 2007).

A síntese de zeólitas a partir de materiais alternativos e de baixo custo teve grande desenvolvimento nas últimas décadas devido ao fato dos processos que utilizam soluções convencionais de alumínio e sílicio apresentarem custos elevados. Sendo assim, a síntese de zeólitas pode ser realizada a partir de qualquer matéria prima que contenha sílica e alumina. No entanto, o uso de cinza de carvão é mais explorado devido à quantidade significativa desse resíduo gerado ao redor do mundo (Izidoro, 2013).

Atualmente a literatura apresenta alguns trabalhos sobre conversão de cinzas em zeólitas por tratamento hidrotérmico alcalino e a sua utilização na remoção de metais em água, mas há poucos estudos empregando-se cinzas geradas no Brasil. O carvão brasileiro apresenta características próprias que irão influenciar as propriedades das cinzas e das suas respectivas zeólitas e, consequentemente, a capacidade de troca iônica do adsorvedor.

Procurando uma maneira de reduzir os custos e simplificar significativamente o processo de produção de zeólita a partir de cinzas volantes, estão sendo desenvolvidos estudos visando a síntese de zeólitas à temperatura ambiente sendo comercialmente mais rentável que os demais processos (Derkowski et al., 2007).

Os estudos deste trabalho visam o reaproveitamento das cinzas de carvão produzidas no Brasil tornando a atividade de exploração do carvão mais sustentável. 


\section{MATERIAIS E MÉTODOS}

A proposta deste trabalho é realizar ensaios de síntese de fases zeolíticas em sistemas abertos, a partir de cinzas de carvão.

A matéria-prima para o trabalho foi constituída de duas amostras. Uma de cinza fina volante e outra de cinza grossa de fundo. As duas amostras foram fornecidas por uma termoelétrica localizada no estado de Santa Catarina.

\subsection{Caracterização das amostras de cinzas de carvão}

As cinzas, grossa e fina foram preparadas por quarteamento em amostras de $500 \mathrm{~g}$.

Para a análise química das cinzas de carvão amostras representativas das cinzas foram caracterizadas pelo método da gravimetria para quantificação do elemento $\mathrm{Si}$, absorção atômica com chama com $\mathrm{C}_{2} \mathrm{H}_{2} /$ ar para quantificar o $\mathrm{Fe}$ e absorção atômica com chama com $\mathrm{C}_{2} \mathrm{H}_{2} / \mathrm{N}_{2} \mathrm{O} /$ ar para quantificar os elementos $\mathrm{Al}$ e $\mathrm{Ca}$. As técnicas de $\mathrm{MEV}$ (microscopia eletrônica de varredura) e difratometria de Raio - X também foram feitas para complementar as análises química das cinzas.

\subsection{Preparo e caracterização das zeólitas sintetizadas a partir das cinzas de carvão}

Procurando uma metodologia que simplifique e torne menos oneroso o procedimento de produção de zeólitas a partir das cinzas de carvão mineral, os experimentos de síntese de zeólitas foram realizados a temperatura ambiente.

Amostras de $100 \mathrm{~g}$ das cinzas de carvão grossa e fina foram tratadas com soluções de $\mathrm{NaOH}$ (VETEC) nas concentrações, razões S/L 1:1 (g/mL) e tempo indicados na tabela 1 . As suspensões foram estocadas em frascos de polietileno e agitadas ocasionalmente. Após o tempo determinado, os sólidos foram separados em papel de filtro, lavados continuamente com água destilada e deionizada até o filtrado atingir $\mathrm{pH}$ aproximadamente 7 , e secos a $60^{\circ} \mathrm{C}$ por 24 horas em estufa. Todas amostras foram levadas para análise de difração de raios-X.

As seguintes variáveis do processo foram testadas:

1) Concentração de $\mathrm{NaOH}(0,5,3$ e 6 mols/L);

2) Tempo reacional. (30, 90, 150 e 300 dias); 
Tabela 1 - Detalhamento dos testes realizados

\begin{tabular}{|c|c|c|c|c|}
\hline Teste & $\begin{array}{l}\text { Massa de cinza }(\mathrm{g}) \\
\text { Fina ou grossa }\end{array}$ & $\begin{array}{l}\text { Volume de } \mathrm{NaOH} \\
\qquad(\mathrm{mL})\end{array}$ & $\begin{array}{c}\text { Concentração } \\
\text { de } \mathrm{NaOH} \\
(\mathrm{mol} / \mathrm{L})\end{array}$ & $\begin{array}{l}\text { Tempo } \\
\text { (dias) }\end{array}$ \\
\hline 1 & 100 & \multirow{12}{*}{$\begin{array}{l}\text { O mesmo volume } \\
\text { de } 100 \mathrm{~mL} \text { de } \\
\text { solução em todo os } \\
\text { frascos }\end{array}$} & 0,5 & 30 \\
\hline 2 & 100 & & 3 & 30 \\
\hline 3 & 100 & & 6 & 30 \\
\hline 4 & 100 & & 0,5 & 90 \\
\hline 5 & 100 & & 3 & 90 \\
\hline 6 & 100 & & 6 & 90 \\
\hline 7 & 100 & & 0,5 & 150 \\
\hline 8 & 100 & & 3 & 150 \\
\hline 9 & 100 & & 6 & 150 \\
\hline 10 & 100 & & 0,5 & 300 \\
\hline 11 & 100 & & 3 & 300 \\
\hline 12 & 100 & & 6 & 300 \\
\hline
\end{tabular}
sintéticas.

A técnica de difratometria de Raio $-X$ foi utilizada para a análise química das zeólitas

\section{RESULTADOS E DISCUSSÃO}

A primeira parte deste estudo consistiu em caracterizar as amostras de cinzas de carvão e posteriormente as zeólitas sintetizadas. Esta análise é importante para se fazer uma comparação entre o material precursor e o produto obtido.

\subsection{Caracterização das amostras de cinzas de carvão}

O potencial de aplicação das cinzas de carvão é definido pela sua composição química. O principal constituinte das cinzas de carvão é a sílica, seguido de alumínio e ferro, indicando que a cinza de carvão está composta principalmente de silicatos de alumínio e ferro ou uma pequena fração de óxidos de ferro (Soto, 2005). Os resultados obtidos para a composição química das cinzas utilizadas são mostrados na tabela 2 abaixo.

Tabela 2 - Caracterização das amostras de cinzas de carvão

\begin{tabular}{|c|c|c|c|c|}
\hline Tipo de cinza & Al & Si & Ca & Fe \\
\hline Cinza fina & $9,6 \%$ & $29,3 \%$ & $0,72 \%$ & $2,9 \%$ \\
\hline Cinza grossa & $9,5 \%$ & $26,5 \%$ & $0,93 \%$ & $5,6 \%$ \\
\hline
\end{tabular}

Através da análise de raios $\mathrm{X}$ foi possível estimar a presença das principais fases minerais presentes nas cinzas utilizadas. As fases que apresentaram picos com maior 
intensidade foram o de quartzo e mulita. As fases magnetita, silamanita, hematita e calcita também estão presentes porém apresentaram picos com menor intensidade, conforme observado nas figuras $1 \mathrm{e} 2$.

Nascimento et al., 2009 e Gross et al., 2007, também identificaram mulita, quartzo e magnetita como principais fases minerais presentes nas amostras de cinza de carvão, características necessárias para o material de partida na síntese de zeólitas.

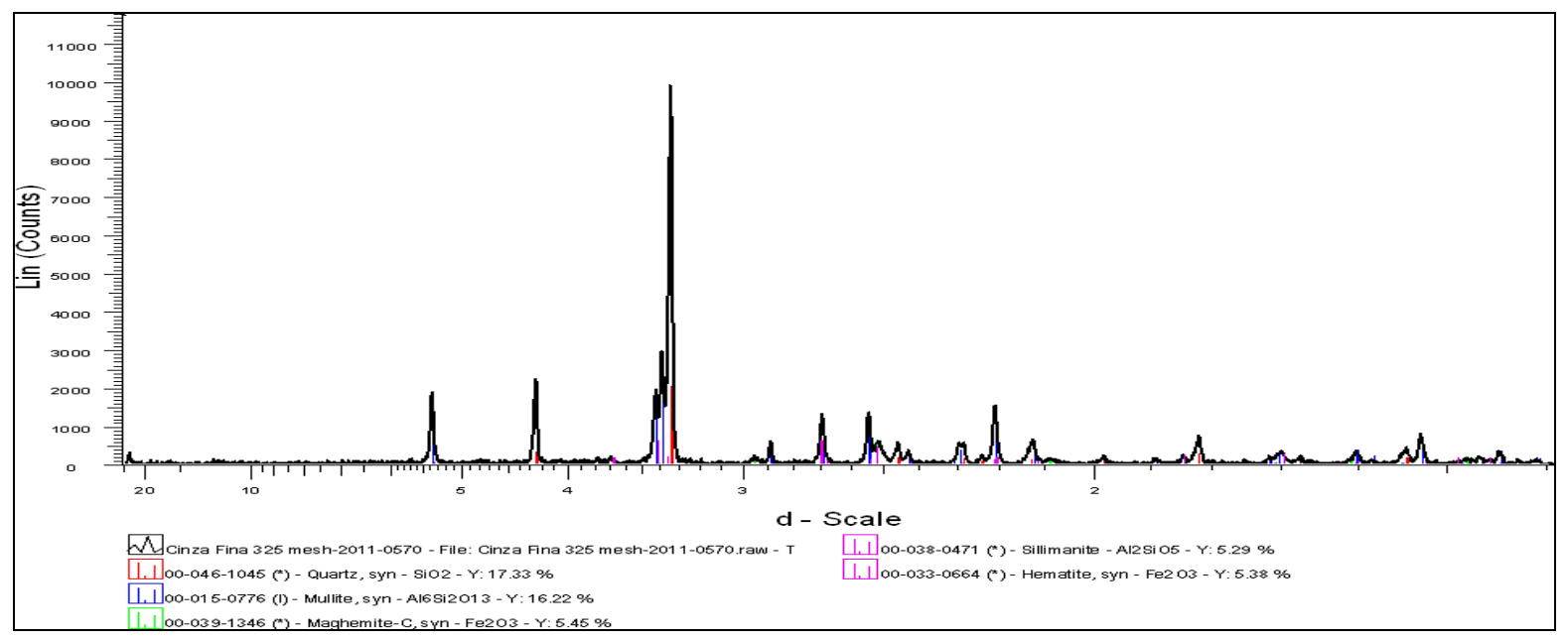

Figura 1- Difratrograma da amostra de cinza de carvão.

\subsection{Caracterização das zeólitas sintetizadas a partir das cinzas de carvão}

Alguns autores, descrevem a transformação de cinzas brasileira em zeólitas utilizando o método da fusão (Isidoro, 2013) ou a conversão hidrotermica utilizando altas temperaturas (Nascimento et al, 2009). Neste trabalho vamos apresentar os resultados obtidos para a sintese de zeólitas utilizando cinzas de carvão mineral brasileira na conversão com temperatura e pressão ambiente.

Na tabela 3 são apresentadas as fases cristalinas encontradas para cada experimento e os principais difratrogramas de raio $\mathrm{X}$ da análise de dados obtidos para os materiais zeoliticos sintetizados estão na figura 2 . 
Tabela 3- Experimentos de zeolitização e fases minerais encontradas

\begin{tabular}{|c|c|c|c|c|c|}
\hline Teste & $\begin{array}{l}\text { Massa de cinza } \\
(\mathrm{g})\end{array}$ & $\begin{array}{l}\text { Volume de } \mathrm{NaOH} \\
\qquad(\mathrm{mL})\end{array}$ & $\begin{array}{c}\text { Concentração } \\
\text { de } \mathrm{NaOH} \\
(\mathrm{mol} / \mathrm{L})\end{array}$ & $\begin{array}{l}\text { Tempo } \\
\text { (dias) }\end{array}$ & $\begin{array}{c}\text { Fases } \\
\text { minerais } \\
\text { presentes }\end{array}$ \\
\hline 1 & 100 & \multirow{12}{*}{$\begin{array}{l}\text { O mesmo volume } \\
\text { de } 100 \text { mL de } \\
\text { solução em todo } \\
\text { os frascos }\end{array}$} & 0,5 & 30 & $\mathrm{Q} ; \mathrm{Mul}$ \\
\hline 2 & 100 & & 3 & 30 & Q; Mul; Phil \\
\hline 3 & 100 & & 6 & 30 & Fau; Q; Mul \\
\hline 4 & 100 & & 0,5 & 90 & $\mathrm{Q} ; \mathrm{Mul}$ \\
\hline 5 & 100 & & 3 & 90 & Q; Mul; Phil \\
\hline 6 & 100 & & 6 & 90 & $\begin{array}{c}\text { Q; Mul; } \\
\text { Fau; Phil }\end{array}$ \\
\hline 7 & 100 & & 0,5 & 150 & $\mathrm{Q} ; \mathrm{Mul}$ \\
\hline 8 & 100 & & 3 & 150 & Q; Mul; Fau \\
\hline 9 & 100 & & 6 & 150 & Q; Mul; Fau \\
\hline 10 & 100 & & 0,5 & 300 & Q; Mul \\
\hline 11 & 100 & & 3 & 300 & Q; Mul; Fau \\
\hline 12 & 100 & & 6 & 300 & $\begin{array}{c}\text { Q; Mul; } \\
\text { Fau; Phil }\end{array}$ \\
\hline
\end{tabular}

(Q: quartzo; Mul: mulita; Fau: faujasita (zeólita X); Phil: philipsita)

Em qualquer intervalo de tempo testados e utilizando altas concentrações de $\mathrm{NaOH}$ (3 e $6 \mathrm{M}$ ), a principal zeólita formada foi a zeólita $X$ (faujasita), além de pequenas quantidades de philipsita. De acordo com a tabela 3 e os difratrogramas de raio X apresentados na figura 2, podemos observar que fases minerais de faujasita foram inicialmente detectadas após 30 dias de contato das cinzas com solução concentrada de $\mathrm{NaOH} 6 \mathrm{M}$. Porém, os melhores resultados, foram obtidos quando se utilizou alta concentração de $\mathrm{NaOH}$ e maior tempo de contato.

$\mathrm{Na}$ síntese de zeólitas a partir de cinzas de carvão utilizando baixa temperatura e pressão foi identificado apenas a formação da fase mineral de faujasita. A presença de resíduos de quartzo e mulita também foram observados (Derkowski et al ., 2007).

A presença de fases não zeolíticas nos produtos da conversão a partir de cinzas de carvão limita a capacidade de uma das principais aplicações das zeolitas qie é a capacidade de troca iônica dificultando as aplicações de zeólitas como adsorvente de metais pesados. (Hui \& Chao, 2006). 


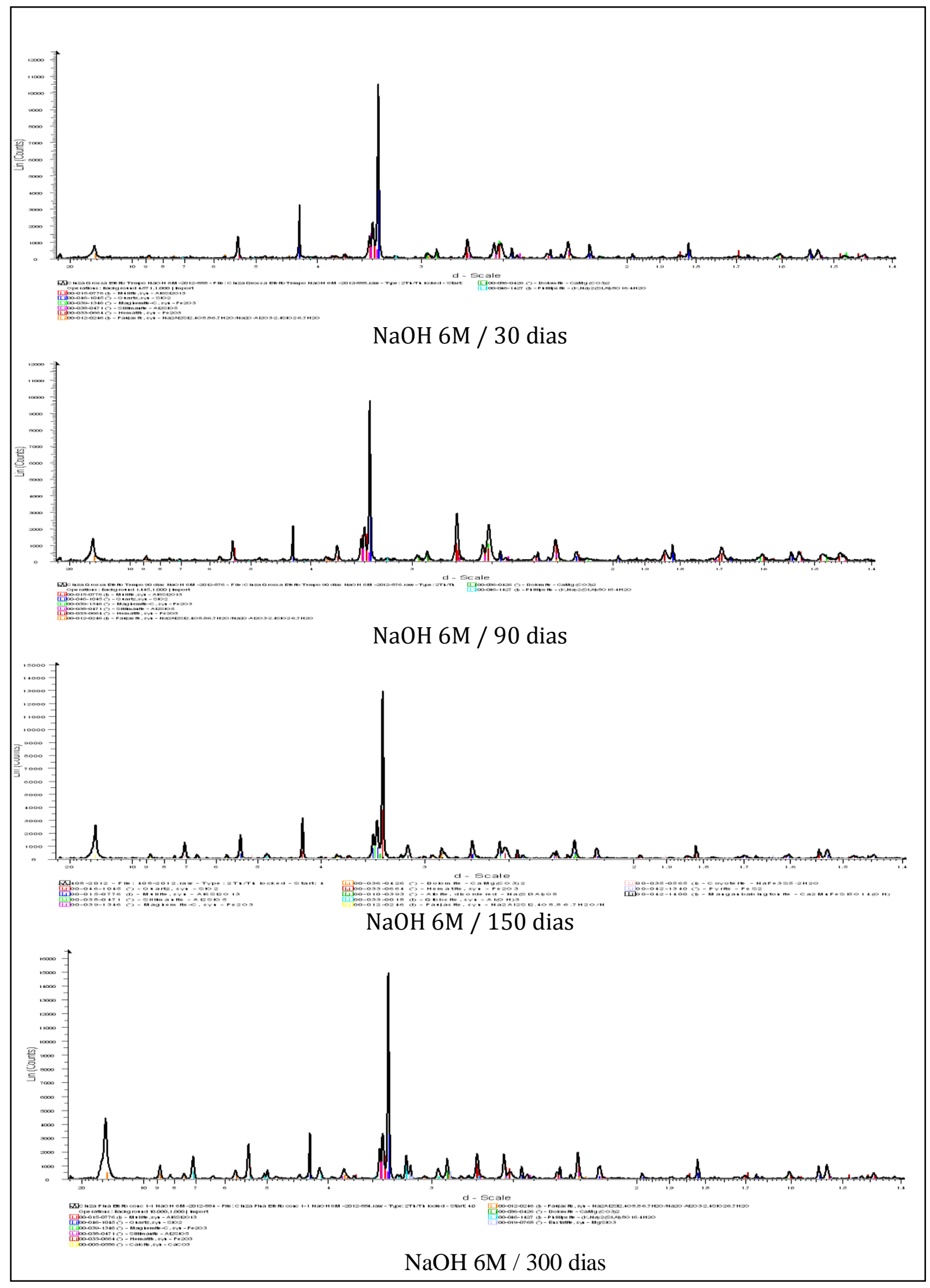

Figura 2 - Difratogramas dos principais materiais zeoliticos sintetizados. 


\section{CONCLUSÃO}

De acordo com os resultados obtidos, podemos observar que fases minerais de faujasita foram inicialmente detectadas após 30 dias de contato das cinzas com solução concentrada de $\mathrm{NaOH} 6 \mathrm{M}$. Porém, os melhores resultados, de acordo com os DRX, para faujasita foram obtidos quando se utilizou alta concentração de $\mathrm{NaOH}(6 \mathrm{M})$ e um maior tempo de contato, como 300 dias, por exemplo.

Através deste trabalho preliminar pode-se concluir que é possível produzir zeólitas por ataque químico com solução de $\mathrm{NaOH}$ a temperatura ambiente a partir de cinzas de carvão. Um aumento do tempo de ataque alcalino bem como um aumento da concentração de $\mathrm{NaOH}$ favorecem um aumento dessa capacidade de síntese zeolítica.

\section{BIBLIOGRAFIA}

DANA J.D., 1981. Manual de Mineralogia (Dana- Hurlbut). São Paulo: Livros Técnicos e Científicos Editora S.A., p.642, 1981.

DERKOWSKI, A., FRANUS, W., WANIAK-NOWICKA, H., CZÍMEROVÁ, A. Textural properties $v s$. CEC and EGME retention of Na-X zeolite prepared from fly ash at room temperature. Int. Journal Mineral Process. v. 82, p. 57-68, 2007.

GROSS, M., SOUlARD, M., CAUllet, P., JOEL, P., SAUDE, I. Synthesis of faujasite from coal fly ashes under smooth tempearture and pressure conditions: A cost saving process. Microporus and Mesoporus Materials. Article in press, 2007.

HUI, K.S., CHAO, C.Y.H. Effects os step-change of synthesis temperature on synthesis of zeolite 4A from coal fly ash. Microporus and Mesoporus materials, v.88, p. 145-151, 2006.

IZIDORO, J.C. Síntese e caracterização de zeólita pura obtida a partir de cinzas volantes de carvão. Dissertação de Doutorado. Ipen - Universidade de São Paulo, 2013.

LORGOUIlloX, G.M., SOUlard, M., CAUllet, P., JOEL, P., MOLEIRO, E. Conversion of coal fly ashes into faujasite under soft temperature and pressure conditions: Influence of additional sílica. Microporus and Mesoporus Materials. Article in press, 2009.

NASCIMENTO, M., SOARES, P.S., SOUZA, V.P. Adsorption of heavy metals cations using coal fly ash modified. Fuel. v.88, p.1714-1719, 2009.

QUEROL, X., MORENO, N., UMANÃ, J.C., ALASTUEY, A., HERNÁNDEZ, E., LÓPEZSOLER, A., PLANA, F. Syntesis of zeolite from coal ash: an overview. International Journal of Coal Geology, v.50, p.413-423, 2002.

SOTO, O.A.J. Remoção de manganês II contido em efluentes líquidos através da sorção em paligorsquita e cinzas de carvão. Dissertação de Mestrado- PUC. Pontifícia Universidade Católica do Rio de Janeiro, 2005. 